\title{
Mars Trace Gas Fluxes: Critical Strategies and Implications for the Upcoming Decade
}

\author{
A White Paper submitted to the \\ Planetary Science and Astrobiology Decadal Survey
}

July 15,2020

\author{
Lead Author: \\ Zachary K. Garvin \\ Princeton University \\ B88 Guyot Hall \\ Princeton, NJ 08544 \\ 443-253-9594 \\ zgarvin@princeton.edu
}

\section{Co-authors:}

Eric Boyd, Montana State University

Melissa Floyd, NASA Goddard Space Flight Center

Rachel L. Harris, Harvard University

Hemani Kalucha, York University

Paul Mahaffy, NASA Goddard Space Flight Center

John E. Moores, York University

Tullis C. Onstott, Princeton University

Haley M. Sapers, USC/JPL/CalTech

Melissa Trainer, NASA Goddard Space Flight Center

\section{Co-signers/Endorsers:}

Sushil Atreya, University of Michigan

Don Banfield, Cornell University

Shawn Domagal-Goldman, NASA Goddard Space Flight Center

Heather Franz, NASA Goddard Space Flight Center

Shane W. Stone, University of Arizona

Kevin D. Webster, Diné College/Planetary Science Institute 


\section{EXECUTIVE SUMMARY}

Measuring trace gas fluxes on the surface of Mars will define new boundary conditions for gas exchange models and life-supporting metabolisms that could produce signatures of extant biological processes. Gas fluxes are the next logical step in evaluating the dynamics of sources and sinks of climate- and astrobiologically-relevant gases within the modern martian system. Such fluxes will build upon current gas abundance data to provide a clearer picture of the atmosphere-surface interactions at higher spatiotemporal scales, while also constraining the crustal contribution to the martian atmosphere over time. Trace gas fluxes can be measured via a joint mobile chamber and static surface station approach using modern detection techniques well-suited for distributed deployment on Mars in the next decade.

\section{INTRODUCTION AND BACKGROUND}

\section{The enigma of martian trace gases}

The past decade of Mars exploration has led to significant advancements in our understanding of the geochemistry and climatology of the planet. Among the outcomes of recent missions is the imperative to determine the composition of the martian atmosphere with higher precision and greater spatial resolution. This goal includes characterizing the abundance of trace gases to reveal vital information about the active processes, climate, and geochemical environments on Mars. Missions such as Curiosity (MSL), Mars Express, and the ExoMars Trace Gas Orbiter (TGO) have independently measured gas concentrations over the course of their ongoing campaigns. Collectively, the reported data has reinforced the critical gaps in knowledge pertaining to trace gas dynamics on Mars. Local and seasonal fluctuations in volumetric mixing ratios for trace gas species $\left(\mathrm{CH}_{4}\right.$ and $\left.\mathrm{O}_{2}\right)$ suggest a seasonal periodicity in specific gas abundances ${ }^{1,2}$ that implies yetto-be-determined processes are controlling their creation, destruction, and exchange. For other trace gas species (e.g., $\mathrm{H}_{2}, \mathrm{CO}, \mathrm{NO}_{\mathrm{x}}$, etc.), such long-term targeted measurements have not been conducted at an equivalent level of detail.

The low abundances of these gases $(<1 \%$ volumetric mixing ratio in the martian atmosphere) present a challenge even for high-sensitivity instruments. The story of $\mathrm{CH}_{4}$ on Mars serves as a representative "case study" in the challenges associated with trace gas detection. Although $\mathrm{CH}_{4}$ trends have been shown to exhibit seasonality as detected by MSL-SAM (Sample Analysis at Mars) at Gale $\mathrm{Crater}^{1}$, it has remained undetected by $T G O$ globally ${ }^{3}$. This conflicting data could suggest a near-surface, fast destruction mechanism for $\mathrm{CH}_{4}$, which would otherwise have an atmospheric lifetime of $\sim 300$ years. However, the sparse trace gas datasets prevent us from definitively identifying the active processes controlling the gas cycling. Discrepancies between analytical platforms operating at different spatial scales highlight the problematic nature of trace gas monitoring on Mars and point toward the need to determine the mechanisms that influence their spatiotemporal dynamics in the atmosphere and surface/subsurface.

\section{Gas Fluxes: the essential next step in trace gas characterization}

The apparent trends in recent trace gas results have set the stage for the next generation of gas studies on Mars - the measurement of gas fluxes. Although compositional measurements provide discrete snapshots of trace gas abundance, flux measurements integrated across time and space deliver more detailed information about the active movement of key gas species and the processes that control them. Flux measurements inherently require higher spatiotemporal resolution data collection methods to produce robust estimates over time. Progressing beyond single timepoint measurements, flux measurements would not only capture surface-atmosphere interactions but provide an additional window into the subsurface, a major unexplored target of the next 


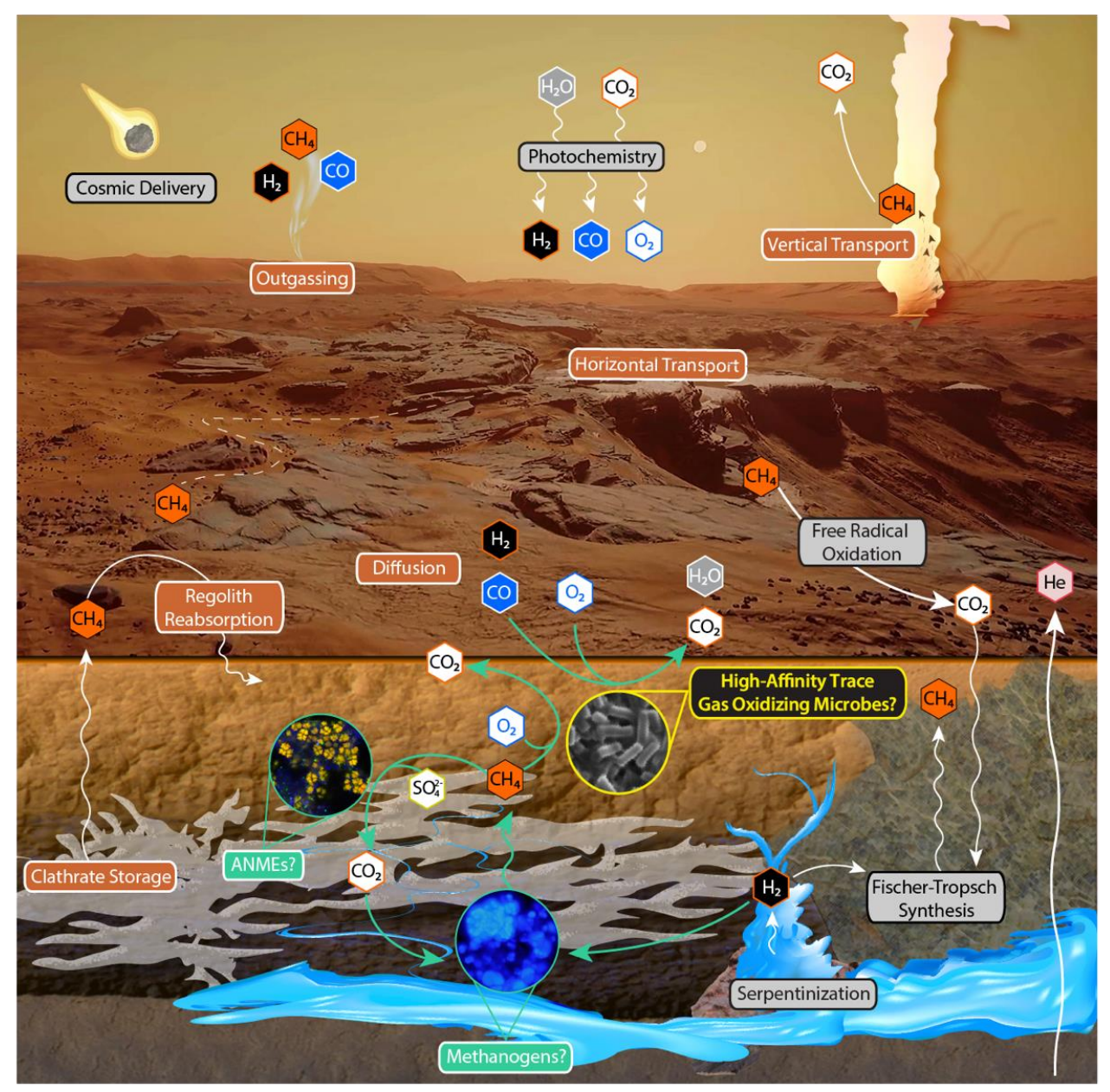

Figure 1. Simplified theoretical gas cycling schematic for Mars driven by gas-metabolizing microorganisms in the shallow subsurface. High-affinity trace gas oxidizing microbes consume photochemically-derived reduced gas species $\mathrm{H}_{2}$ and $\mathrm{CO}$ coupled to $\mathrm{O}_{2}$. These microbes along with anaerobic methanotrophs (ANMEs) also oxidize $\mathrm{CH}_{4}$ produced from geochemical reactions and methanogenic organisms. Adapted from Dr. R. Harris, Harvard University.

generation of Mars missions with significant potential and relevance for life detection. Additionally, information derived from trace gas fluxes would help to characterize gas cycling on the planet, indicating the potential abiotic and/or biotic processes that control them. Greater understanding of these interactions and processes will dramatically improve modeling the evolution of the atmospheric and climatic systems on Mars by constraining boundary conditions. The fuel for extant life on Mars?

One of the highest priority justifications for trace gas flux measurements are their implications for biological activity. The coupling of reduced gas species (e.g., $\mathrm{CH}_{4}, \mathrm{CO}, \mathrm{H}_{2}$ ) with a strong oxidant represents a steep, energy-rich redox gradient that can be harnessed by microorganisms. Microbial trace gas oxidation has become increasingly recognized as a significant metabolic feature that supports life in a variety of oligotrophic terrestrial environments, including past and present Mars analogue sites such as Atacama Desert volcanic fumaroles ${ }^{4}$, Canadian High Arctic cryosols $^{5}$, Antarctic Dry Valley soils ${ }^{6}$, cavern soils ${ }^{7}$, and Andean Altiplano arid saltpans ${ }^{8}$. Chemolithotrophs in these soil environments are capable of utilizing these reduced gases at ambient atmospheric concentrations (often parts per billion, ppbv, scale). On Mars, similar 
metabolic strategies could have evolved over time as water availability and gas sources diminished on the martian surface, introducing selective pressure to adapt to lower, ambient concentrations of atmospheric gas reductants and potentially oxidants (i.e., $\mathrm{O}_{2}$ for aerobic metabolisms). This scenario implies that trace gas consumption could remain a prevalent tactic for modern extant life on Mars despite the low abundances of reduced gas species in the atmosphere (Figure 1). Some trace gas-oxidizing microorganisms utilize perchlorates as oxidants in their energy metabolisms ${ }^{9}$, further strengthening their potential relevance to Mars where perchlorates have been detected on the surface ${ }^{10}$. As on Earth, the significance of the role of these metabolisms in gas cycling dynamics at the ecosystem or global scale may be currently underestimated for Mars. Through the measurement of soil gas fluxes, hot spots of gas uptake could be detected and used as a proxy or biosignature of extant life in the surface and shallow subsurface of Mars.

\section{GOALS AND RELEVANCE}

We broadly define the quantification of gas fluxes as a primary science goal for Mars exploration in the next decade. Trace gases including reduced species (i.e., $\mathrm{CH}_{4}, \mathrm{H}_{2}$, and $\mathrm{CO}$ ) as well as $\mathrm{O}_{2}$ and $\mathrm{He}$ are particular targets of interest due to their relevance to climate, biology, and the subsurface (e.g., geochemical systems). Importantly, fluxes provide insight into both the magnitude and directionality of changes in gas composition, which can be measured at extremely fine scales and with high sensitivity. Thus, gas flux should be investigated across the two major variables of space and time to build upon current abundance data and improve understanding of the active mechanisms of gas exchange across the planet. Finally, measurement of the stable isotopic composition of the trace gases remains a high priority as it can further constrain their origin and the processes resulting in their change in concentration.

Measurement of gas fluxes is the logical next step in characterizing the martian atmosphere, surface, and subsurface from the perspectives of astrobiology and climatology. Knowledge of gas fluxes would further develop multiple objectives outlined in the 2013-2022 NAS Planetary Science Decadal Survey ${ }^{11}$. Mapping the distribution of trace gases, as is being performed by $T G O$, is identified as a direct approach to detect regions of active subsurface activity and search for extant life at broad, regional scales. In-depth understanding of martian trace gases can help to determine the roles of known and unknown processes (e.g., geochemical, photochemical, biological) in controlling the current atmospheric composition to better constrain and refine relevant models. Evidence of trace gas uptake into the (sub)surface can also be a potential indicator of microbial metabolic activity, as noted in the updated 2020 MEPAG goals ${ }^{12}$.

Deriving gas fluxes expands upon the regional timepoint measurements and global scale mapping efforts achieved thus far and provides more consistent monitoring for a high-resolution view of gas exchange between different reservoirs. Perhaps most significantly, fluxes can capture the spatial and temporal variation in these exchanges in terms of both gas abundance and rates of movement. As the MEPAG goals explicitly state, our knowledge of trace gas sources, sinks, and abundance across space and time remains far too limited ${ }^{12}$. By bolstering the existing gas concentration data with flux (and potentially isotopic) measurements, we can continue to piece together the story of Mars as a connected system through the lens of gas transport.

\section{SCIENCE OBJECTIVES}

We outline three primary science objectives and associated measurements/experiments for studying trace gas fluxes on Mars in the upcoming decade:

\section{Objective 1: Spatial Characterization}


Flux measurements should be performed via a targeted approach to complement the atmospheric gas compositions that have been assessed regionally and globally. Comparatively, more "micro-scale" analyses focused on the atmosphere-surface boundary would provide an "ecosystem" level view of Mars through the combination of targeted $\left(\mathrm{m}^{2}\right)$ and local $\left(100 \mathrm{~m}^{2}\right)$ scale characterization. By collecting flux data across varying degrees of spatial separation, the general distance(s) at which changes in flux occur from one site to another can be determined. Significant disparities in flux would reveal whether gas exchange is more dominantly controlled regionally or at smaller scales within a given locality, influenced by the underlying geology.

Comparing local fluxes to average regional fluxes (e.g., from $T G O$ ) would provide insight into the heterogeneity of gas exchange across different spatial scales. Targeted experiments would identify specific locations of gas "hot spots" where flux (uptake and/or emission) is particularly elevated within a given monitored region. A key sub-objective is to compile geographical, meteorological, geochemical, isotopic, and geological characteristics of zones demonstrating active gas exchange to determine common environmental features.

\section{Objective 2: Temporal Variation}

Given the fluctuations of some trace gases that have been recently reported for the modern martian atmosphere, establishing the ability to measure their fluxes consistently and with high frequency should be a major priority for the next generation of Mars missions. Long-term, continual monitoring of gas flux at local scales would provide a more nuanced view of the apparent periodicities in atmospheric abundance, helping to confirm and expand upon current data indicative of seasonal variation in $\mathrm{CH}_{4}$ and other diagnostic trace gas species. The consistent collection of time series data at the surface-atmosphere boundary would allow for more precise characterization of trends and the rates with which gas is regularly exchanged.

A critical question is whether the purported seasonal variations in $\mathrm{CH}_{4}$ and $\mathrm{O}_{2}$ are the most significant temporal signal on Mars or whether trace gases exhibit more dramatic fluctuations at other temporal scales. For instance, a strong diurnal cycle could be the dominant source of gas variation due to the magnitude of temperature swings over the course of a sol. In the specific case of $\mathrm{CH}_{4}$, Moores et al. (2019) determined that diurnal variations in the planetary boundary layer (PBL) could account for the discrepancies between $T G O$ and $S A M$ measurements ${ }^{13}$. The described models indicate that nighttime suppression of the PBL could allow $\mathrm{CH}_{4}$ to accumulate near the surface, leading to the elevated $\mathrm{CH}_{4}$ concentrations measured by $S A M$ at circum-midnight timepoints. Alternatively, abundances may be controlled by more irregular or stochastic fluxes without clear trends at shorter timeframes, as suggested by the persisting $\mathrm{CH}_{4}$ plumes observed by $S A M$ at week-scale durations. In either scenario, the measurement of high-resolution flux data would better define these trends to improve and constrain models.

\section{Objective 3: Modeling and Implications for Life}

Rigorous investigative efforts to accomplish Objectives 1 and 2 would provide a new framework for theorizing about metabolic strategies for potential microbial life and its ability to thrive on Mars. Gas fluxes have been invoked in the past to estimate theoretical biomass limits that could be supported by modern martian environments. Weiss et al. (2000) ${ }^{14}$ proposed a potential global biomass limit of $\sim 10^{10} \mathrm{~kg}$ based solely on aerobic metabolisms relying on the diffusion of photochemically-sourced $\mathrm{CO}$ and $\mathrm{H}_{2}$ down to $10 \mathrm{~m}$ depth. Sholes et al. (2019) ${ }^{15}$ built upon this work, estimating a limit of $\sim 10^{11} \mathrm{~kg}$ global biomass to a maximum $10 \mathrm{~km}$ depth (Figure 2) by optimizing unknown model parameters and including $\mathrm{H}_{2}, \mathrm{CO}$, and $\mathrm{CH}_{4}$ metabolisms. 


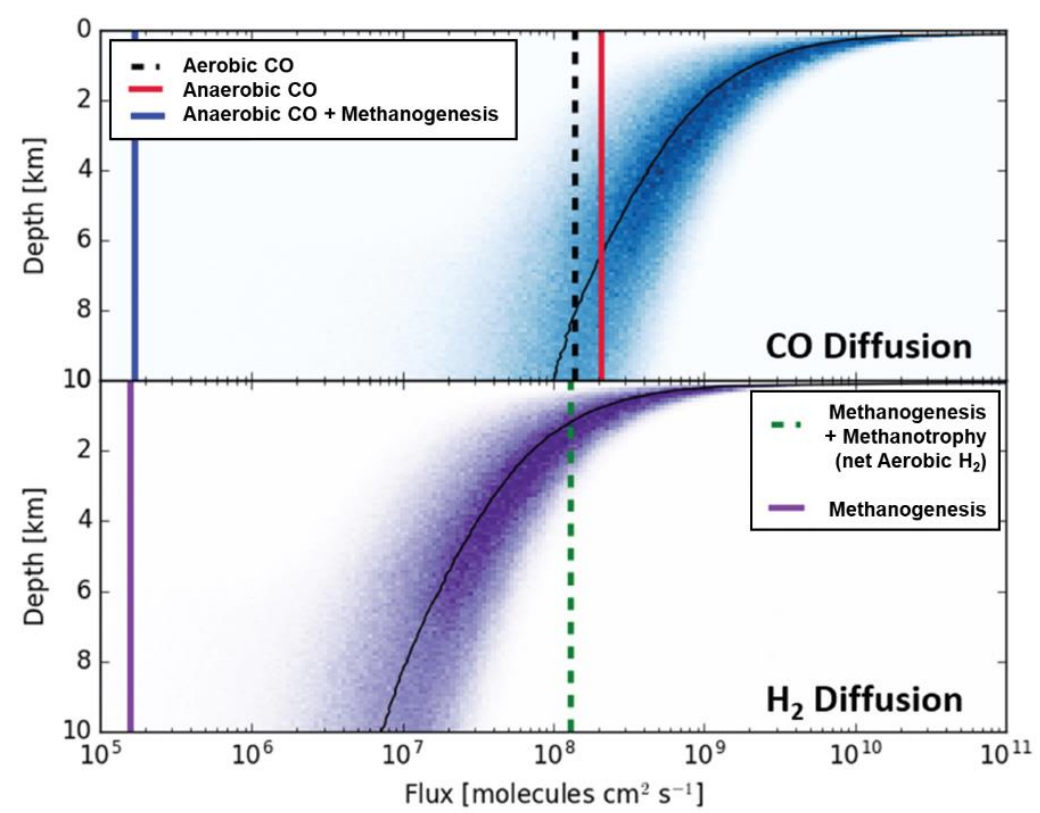

Figure 2. Estimated fluxes of photochemical $\mathrm{CO}$ and $\mathrm{H}_{2}$ into the subsurface via diffusion using Monte Carlo simulations (black curves). Vertical lines represent modeled maximum fluxes due to microbial trace gas metabolisms (assumed 100\% efficiency). Intersection points represent shift from biological limitation by photochemical rates to limitation by diffusive flux. Models and figure adapted from Sholes et al. (2019).

has thus far limited the value of such modeling endeavors. Employing the proposed targeted approach to flux measurements, more specific regional models could be developed for assessing biological potential in locations identified as hot spots of gas activity. These sites could represent areas where life is actively participating in gas cycling or where it is most likely to be taking advantage of redox gradients established by abiotic physical processes. Thus, the flux data could serve as a guide for further astrobiology targets and focused experiments. Correlations between environmental parameters and gas fluxes would also have significant implications for biology, as conditions promoting gas flux may translate to life-favorable settings. By combining this information with follow-up measurements of the stable isotopic signature of the trace gas and existing knowledge of rates for inorganic processes including atmospheric photochemistry and geochemical reactions, the probability of extant biological activity could be defined.

\section{PRIORITY TARGET SITE TYPES}

As atmosphere-surface interactions are relevant globally across the surface of Mars, there is a large degree of flexibility in choosing a site for trace gas flux measurements. Regions containing active or previously active systems characteristic of gas cycling are a natural target of interest. Potentially recent volcanic sites, exposed caverns, subsurface ice, and ancient hydrothermal systems are particularly enticing candidates due to their high rates of gas emission and association with reduced trace species derived from the subsurface. Trace gas-oxidizing organisms are prevalent in analogous terrestrial environments, indicating such havens could exist for similar life on Mars. Zones of distinct environmental gradients are another practical target for diverse 
measurements and experimental design. Geological, geochemical, and meteorological gradients are ideal for comparison studies at local spatial scales, which would enable flux to be explored across a range of specific variables without requiring a vast network of instrumentation across the martian surface or long-distance mobility. Revisiting previously explored regions could also be beneficial for the purpose of building upon preexisting data. Candidate areas identified via past missions as exhibiting local fluctuations in trace gas abundance and, thus, having potentially active processes would lower mission risks given variation in trace gases is already known.

\section{MISSION STRATEGIES}

The proven track record of trace gas measurements on Mars combined with widely applied techniques for robust detection (sensitivity) and flux determination in terrestrial environments make this a highly achievable mission concept for development in the next decade. A successful mission prioritizing trace gas fluxes will require (1) infrastructural equipment to conduct flux experiments and monitoring, (2) instrumentation to measure gases with high sensitivity and signalto-noise, and (3) vehicle support to enable sampling flexibility in measurement locations.

\section{(1) Infrastructural Equipment}

On Earth, surface gas fluxes are typically measured by either soil flux chambers or eddy covariance methods. Flux chambers target relatively small surface areas (e.g., $20 \mathrm{~cm}$ diameter dome creating a closed system between the regolith and enclosed atmosphere). A key advantage to this technique is portability due to their small size, low weight, and ease of deployment/set-up. As a result, they can be repeatedly redeployed to multiple locations within a region for short-term experiments. For a martian surface mission, this would be valuable for fitting payload restrictions as only a single lightweight chamber is needed. Another advantage of flux chambers is that trace gas species may accumulate over time, enabling concentrations to be more accurately determined than would be the case for a direct atmospheric sample. Eddy towers provide another approach by measuring surface gas fluxes that capture boundary level atmospheric processes ${ }^{16}$. Once deployed, the tower can remain as a static monitoring station for long-term measurements. It would thus be advantageous to employ a hybrid approach on Mars to best accomplish the proposed objectives.

\section{(2) Detection Instrumentation}

High-frequency detection methods are required for accurate gas flux calculations to maximize measurements per unit time. Currently, SAM measurements represent the highest temporal resolution data collected for local gas abundance on the martian surface with a measurement frequency of once per day. As established in Objective 2, determination of active processes and consistent trends will require more regular timepoints with multiple measurements over a diurnal cycle. Direct optical spectroscopy techniques are the standard for achieving real-time, rapid measurements while maintaining high specificity and signal-to-noise ratios. Off-axis integrated cavity output spectroscopy (OA-ICOS) offers ideal performance for consistent, high-precision trace gas measurements. Though slightly less sensitive than other cavity enhanced methods such as cavity ringdown spectroscopy (CRDS), ICOS maintains the trace detection capabilities $(<1$ ppb) needed to capture small changes in low-abundance trace species like $\mathrm{CH}_{4}$ while improving upon spectral range and robustness for field deployment. Among trace gases of interest, He and homonuclear diatomic species such as $\mathrm{H}_{2}$ and $\mathrm{O}_{2}$ are not directly detectable by these optical methods due to lack of adsorption bands. Chemical sensors (such as palladium-based methods for $\mathrm{H}_{2}$ ) are a potential substitute, maintaining high frequency at the loss of some sensitivity. For high sensitivity detection, alternative techniques such as gas chromatography (GC) are required to separate the gases prior to detection via UV/fluorescence after ionization or by coupling to a 
suitable mass spectrometer (MS). An important (though not essential) feature of these techniques is that they are capable of determining isotopic ratios of the gas species.

\section{(3) Vehicle Support}

To address Objective 1 and take full advantage of flux chamber portability, a Mars mission using this technique would require horizontal mobility to vary the positions of the chamber. A small MER-class solar-powered rover is a plausible option for carrying a flux chamber short distances across nonhazardous terrain for multiple surface deployments. Though relatively slow, a rover would optimize mass allocations needed for detection instruments that would require deployment along with the chamber itself. Alternatively, advancements in rotorcraft technology (i.e., beyond that of the Mars Helicopter demo on the Mars 2020 mission) could be utilized to transport a flux chamber over longer distances $(\sim 1 \mathrm{~km}$ range). Though more restrictive in terms of payload, rotorcraft would significantly improve the breadth of experiments that could be conducted by a single chamber by decreasing travel time and increasing the diversity and number of potential measurement sites.

\section{CONCLUSION}

Trace gas fluxes on Mars represent a key gap in measurements relevant to the highest priority climate and extant life goals established by MEPAG and in previous Decadal Surveys. Recent findings from the Curiosity SAM suite and $T G O$ suggest that fluxes are critical for unravelling the details of gas sources and sinks, as well as key transport mechanisms. Using the three primary objectives summarized herein and the conceptual implementation possibilities, fundamental trace gas fluxes can be measured within the next decade synchronous with planned Mars surface missions using well-established techniques that directly support both future astrobiology goals and the ongoing ambition to understand Mars as an active system.

\section{REFERENCES}

$1 \quad$ Webster et al. (2018). Science. 360, 1093-1096. doi: 10.1126/science.aaq0131

2 Trainer et al. (2019). J. Geophys. Res. Planets 124, 3000-3024. doi: 10.1029/2019JE006175

$3 \quad$ Korablev et al. (2019). Nature 568, 517-520. doi: 10.1038/s41586-019-1096-4

$4 \quad$ Lynch et al. (2014). Front. Microbiol. 5, 1-13. doi: 10.3389/fmicb.2014.00698

$5 \quad$ Lau et al. (2015). ISME J. 9, 1880-1891. doi: 10.1038/ismej.2015.13

$6 \quad$ Ji et al. (2017). Nature 552, 400-403. doi: 10.1038/nature25014

7 Webster et al. (2016). Chem. Geol. 443, 1-9. doi: 10.1016/j.chemgeo.2016.09.020

$8 \quad$ Garvin et al. (2019). AGU Fall Meet. 2019

9 Myers \& King (2017). Front. Microbiol. 8, 1-8. doi: 10.3389/fmicb.2017.02571

10 Hecht et al. (2009). Science. 325, 64-67. doi: $10.1126 /$ science. 1172466

11 Committee on the Planetary Science Decadal Survey (Washington, D.C., 2011).

12 MEPAG (2020) (Mars Exploration Program Analysis Group (MEPAG): 2020). at <https://mepag.jpl.nasa.gov/reports.cfm>

13 Moores et al. (2019). Geophys. Res. Lett. 46, 9430-9438. doi: 10.1029/2019GL083800

14 Weiss et al. (2000). Proc. Natl. Acad. Sci. 97, 1395-1399. doi: 10.1073/pnas.030538097

15 Sholes et al. (2019). Astrobiology 19, 655-668. doi: 10.1089/ast.2018.1835

16 Wille et al. (2008). Glob. Chang. Biol. 14, 1395-1408. doi: 10.1111/j.13652486.2008.01586.x 